\title{
Gametophytic self-incompatibility or the more things change...
}

\author{
David L. Mulcahy and \\ Gabriella Bergamini Mulcahy
}

In 1983, we suggested a particular interpretation of gametophytic self-incompatibility. It differed from the widely accepted interpretation in several regards. The first of these was that our model is a version of the complementation model. That model indicates that the metabolic qualities of pollen tubes and styles in which they grow must "complement" each other. Otherwise pollen tubes fail to reach the ovules in time to accomplish fertilisation. Failure of pollen tube growth is thus passive. The classical model indicates that pollen tubes carrying specific genetic factors trigger the release of pollen inhibiting substances and are thereby opposed. In this, the classical "oppositional" model, failure of pollen tube growth is the result of active processes. Our version of the complementation model, termed the "heterosis" model because it indicates that pollen-style interactions mimic the heterotic interactions between parental genomes in a hybrid, differs from the oppositional model in a second respect. The heterosis model indicates that incompatibility is determined by polygenic systems, each locus being oligoallelic (2-10? alleles). The classical model indicates that determination of self-incompatibility is oligogenic (1-4 loci) with each locus being polygenic ( $2-50+$ alleles). Other distinctions between the two interpretations are consequences of these basic differences.

Lawrence, Marshall, Curtis, and Fearon (1984), hereafter cited as "LMC\&F", first object to our paper on the basis of Lewis' (1954) point that, since every style contains two different $S$ alleles, any pollen grain will complement at least one of them. Complementation models are therefore impossible. This, of course, assumes that there is only one incompatibility locus segregating and that each incompatibility allele is codominant in the style. If these assumptions are correct, complementation models are indeed conceptually impossible. However, we make neither of these assumptions. In fact, our model involves several
Department of Botany, University of Massachusetts, Amherst, MA 01003 USA.

loci, and alleles which influence incompatibility are expressed in the style only when they are homozygous recessive.

The second point raised by LMC\&F in their introduction is that, "Each individual would need to produce a complementary substance for every allele present in the population that is not carried by itself; etc.". By way of explanation, how do we interpret the fact that some hybrid zygotes live and grow while others die? We do not speculate on whether this reflects the operation of "oppositional" or "complementary" substances. The heterosis model suggests that, "self-incompatibility is perhaps merely one aspect of extensive pollen-style interactions." Thus we suggest that there are, in fact, no, "complementary substances," that is, none which functions only in the incompatibility reaction.

LMC\&F then take issue with all five of the points that we cite as inconsistent with the oppositional model. LMC\&F object that our model predicts a high frequency of self-compatible or pseudo self-compatible plants. They also reject our suggestion that the classical model does not adequately explain the inverse correlation which exists between the strength of the incompatibility reaction and the level of stylar heterozygosity. (Larsen (1977) reported that increasing frequencies of heterozygosity at incompatibility loci in Beta vulgaris (four incompatibility loci) reduces the strength of the self-incompatibility reaction. Lundqvist (1958) reported similar findings in rye (two loci), and we assume that the same hypothesis should apply to single locus systems.)

The first part of our response is that, since heterozygosity is the normal state for self-incompatibility loci, (see studies by Atwood, 1944; Williams, 1947; and Fearon, Hayward, and Lawrence, 1983), both the classical and heterosis models predict that pseudo self-compatibility should be common. (A later quote from East (1927) emphasises 
the high degree of pseudo self-incompatibility.) More to the point is what frequent pseudo selfincompatibility would indicate: According to the classical model, the $S$-locus is extremely ancient and so highly favoured by natural selection as to have permeated the angiosperms from one or a few evolutionary origins (see Whitehouse, 1950; Lewis and Crowe, 1958). If this assumption is correct then surely natural selection would also have favoured the development of self-incompatibility alleles which function effectively in the heterozygous condition (their natural state). Since this has not happened, we wonder if self-incompatibility is really the expression of an ancient, strictly conserved, and nearly monophyletic locus. The heterosis model, in contrast, indicates that self-incompatibility is a dynamic phenomenon, characterised by quantitative variation and capable of multiple origins.

Another piece of evidence that we cited was the generation or activation, by inbreeding, of new $S$-alleles (see Gastel and Nettancourt, 1975). In fact one plant exhibited 5 different specificities in the style. That is, it rejected pollen of 5 different $S$-allele types. More surprising still was the fact that none of the new specificities was expressed in the pollen. That is, the pollen of these inbred plants was accepted by plants which, in the reciprocal cross, were incompatible pollen donors. Furthermore, some new $S$-alleles appeared more often than did others. Also, when the inbred plants were outcrossed or backcrossed, the new specificities usually vanished. LMC\&F are obviously and understandably reluctant to accept these extraordinary findings, especially in view of a subsequent study by Sree Ramulu (1982). He also inbred individuals of Lycopersicon peruvianum but, unlike Gastel and Nettancourt, obtained no new $S$-alleles. Furthermore, in a "more thorough analysis", he was unable to confirm the presence of new specificities in the very plants which Gastel and Nettancourt had produced by inbreeding. Finally, when $S$-alleles of the original material were put into a different genetic background and then, once again, inbred, they failed to generate new specificities

In attempting to disentangle this rather bewildering data set, we remind the reader that the heterosis model indicates that incompatibility results from pollen carrying deleterious recessive alleles for which the style is homozygous. Thus we suggest that the inbreeding accomplished by Gastel and Nettancourt generated enough homozygous loci in the style so that several pollen genotypes, for example, $S_{1}, S_{2}, S_{3}, S_{5}$, and $S_{7}$, were unable to penetrate those styles. This would be interpreted as the activation, by inbreeding, of these $S$-alleles in the style. However, when pollen of the inbred plants was applied to $S_{3}, S_{5}$, and $S_{7}$ containing styles, the pollen tubes were able to penetrate, indicating that the new specificities were not expressed in the pollen. We suggest that these results would be obtained if the plants used to test pollen of the inbred plants were not themselves inbred (see our fig. 3). Thus they supported pollen from inbred plants. As for the fact that particular specificities appear more often than do others, this probably means that the deleterious alleles which relate to those types are more common than are others. Furthermore, when inbreds are outcrossed, the level of heterozygosity of the progeny is raised and the new specificities vanish. Sree Ramulu also inbred $L$. peruvianum, but the fact that he obtained no new specificities indicates only that his material was relatively free of deleterious recessives which would make new specificities possible. In effect, we are generally not surprised when some genotypes are depressed by inbreeding and others not at all. The present issue is no different from that.

As for the "more thorough analysis" referred to by LMC\&F, it was presumably "another analysis" which is a very different statement. If the pollen sources used as testers by Sree Ramulu were in any way genetically different from those used by Gastel and Nettancourt, and if they had fewer alleles in common with $006-S_{1} S_{2}$ clone than did the original testers, it is not at all surprising that results different from the original were obtained. When hybridised with other lines, or backcrossed, entirely new sets of compatibility relationships would be generated. (See figs. 1 and 4, Mulcahy and Mulcahy, 1983.)

Before leaving this point, we must emphasise that there are two compelling reasons why the Gastel and Nettancourt work must not yet be dismissed. Firstly, there is certainly not convincing evidence against it. Secondly, as we suggest below, the issue of the number of incompatibility alleles found in natural populations is far from resolved. If mutation will not provide new $S$-alleles (see Lewis, 1954), what is their source if not that indicated by Gastel and Nettancourt?

The third point that we raised was the difficulty of mapping the $S$-locus. We agree with Nettancourt (1977) that the best attempt has been that of Martin (1961). This value judgement obviously reflects the fact that, unlike most self-incompatible plant 
material, one of the species involved, Lycopersicon esculentum is very well mapped. Martin found that, "Self-incompatibility appears to be controlled by two or more dominant genes." (Italics ours.) Nettancourt also suggests that these data do not demonstrate that the $S$-locus is directly involved in the self-incompatibility character found to segregate in the F2 and backcross progenies.

Another piece of evidence that we cited was that the total number of $S$-alleles is so great. $\mathrm{LMC} \& \mathrm{~F}$ dismiss this issue with two points. Both they (Campbell and Lawrence, 1981, Papaver rhoeas) and Emerson (1939, 1940), dealing with Oenothera organensis, find that most of the $S$ alleles occurred more than once in population samples. However, we should examine these two studies carefully. Oenothera organensis is well known to be relict, the entire species consisting of no more than a few thousand individuals. It should thus hardly be expected to exhibit the degree of genetic variation which typifies most species. As for Papaver rhoeas, Campbell and Lawrence (1981) report that the material consisted of eighteen families from three different localities. "Each family has been obtained by one to four generations of sib matings among the descendants of a single non-inbred family of full-sibs cilosen at random from the families of an intra-locality." (Italics ours.) In the same paper, they state, "Though none of the semi-inbred families had originated from the same pair of ancestors as another, some were drawn from the same male or female array in which case their ancestors were related as half-sibs." We assume that such steps are necessary to prepare material which is appropriate for analysis, but we also wonder how much genetic variation is lost in one to four generations of sib matings, semi-inbred families, etc. Aside from the above two studies, the only two extensive surveys of $S$-alleles within natural populations are those of Atwood (1944) and Williams (1947). Williams found that 20 plants of Trifolium pratense contained 37 different $S$-alleles, 34 of these represented only once and 3 of them twice. In a second sample, 24 plants contained 41 different $S$-alleles, 34 found once, and 7 twice. Atwood obtained strictly comparable results. Thus, in both cases it was found that most allelles occurred only once, and therefore the number of $S$ alleles must be very great. Campbell and Lawrence (1981) apparently dismiss the observations of Williams, in part, because they are based on seed set rather than observations of pollen tubes and, in part, on the fact that "there was no attempt to cross-classify the alleles from one population with those of another". Fearon, Hayward, and Lawrence (1983), however, make heavy, though not exclusive, use of seed set data themselves, and, if we consider the two populations separately, there is no reason to cross-classify, although the data obtained would be interesting. We thus find no reason to doubt that the number of $S$-alleles present in natural populations is very great. With a single $S$-locus, genetic drift should quickly deplete much of this allelic variation, since each allele would be carried by only a few individuals, and yet, this has not occurred. However, with the multiple locus heterosis model we suggest, each locus would carry only a few alleles, the effects of drift would become insignificant, and the richness of compatibility reactions would persist as observed.

On a different aspect of $S$-allele numbers, LMC\&F also point out that Oenothera organensis, is composed of 5000 rather than 500 plants, as once thought. In fact, Campbell and Lawrence (1981) state, "Thus, after 40 years, the problem of the number of self-incompatibility alleles in the Oenothera population appears at last to have been solved." However, Wright (1939) explained that the number of $S$-alleles which could be maintained in a population is determined by both the size of the population and the mutation rate at the S-locus. The size of the Oenothera organensis population is now known, but that is only one part of the information we need. The other part, the rate with which new $S$-alleles are produced by mutation (or other means) is absolutely unknown. Wright (1939) reasonably speculated that it might be on the order of $1 \times 10^{-5}$ or $1 \times 10^{-6}$ and, on that basis, a population of 5000 plants could contain up to $40-50$ different $S$-alleles, about the number reported in Oenothera organensis. This, presumably, is the source of the statement by Campbell and Lawrence (1981). However, the only data known to us on the rate of mutations at the $S$-locus are those of Lewis $(1951,1954)$. He stated, “... no mutation from one operative $S$ allele to another has been found out of a total of $350 \times 10^{6}$ tested".

We must thus assume that new operative alleles appear at a rate lower than 1 in $350 \times 10^{6}$. If Lewis had found one new $S$-allele in his sample, and we were to accept this as the rate with which new alleles appear, this rate of mutation, according to fig. 5 in Wright (1964), would be sufficient to maintain approximately $35 S$-alleles in a population of 5000 plants. However, Lewis did not find a single new $S$-allele in his sample. Therefore, according to the classic model, we must assume that the maximum number of $S$-alleles allowed in a popula- 
tion of 5000 plants is less than 35 . In any case, since the rate of mutation at the $S$-locus is unknown, the problem of the number of $S$-alleles in Oenothera organensis or in any other species, for that matter, is not solved.

The fifth point that we raised was based on a study by Hoffman and Wenzel (1981). They hybridised self-incompatible and self-compatible lines of rye (not ryegrass, as we erroneously stated) and obtained a self-compatible hybrid. Due to a typographical error we indicated that the hybrid was self-incompatible, but, fortunately this did not modify the concept being considered. Irregardless of which condition was dominant, the hybrid was heterozygous for compatibility and incompatibility. Fourteen dihaploids were regenerated and, of these, 10 were self-compatible, 4 self-incompatible. On the basis of this, we reported that regenerated plants contained a high frequency of self-compatible individuals. However, we have since learned that, when the number of regenerated individuals was increased, the proportions of compatible and incompatible individuals were very nearly equal. We therefore withdraw the fifth point of our original paper.

Beyond disagreeing with the five points we originally raised, $\mathrm{LMC} \& \mathrm{~F}$ also state four other criticisms of the heterosis model. The first of these, too many "complementary substances", has already been discussed. Considering next the fourth and the second criticisms of our model (see our figs. 3 and 4), LMC\&F are concerned with the fact that we arbitrarily assumed that a score of -8 or lower results in incompatibility. We should have emphasised what we assumed was the fairly obvious point, namely that there is no reason to consider that all alleles at all loci have precisely equal effects on pollen tube growth rates. Our point in presenting figs. 3 and 4 related to the observations that increasing stylar homozygosity intensifies the incompatibility reaction and also activates new incompatibility specificities. We maintain that the oppositional model accommodates the first observation with difficulty (the limited precursor concept) and the second observation (see Gastel and Nettancourt, 1975), not at all. The heterosis model is conceptually capable of accommodating both sets of observations.

The second criticism raised by LMC\&F was that our model is incapable of predicting what happens in practice. We suggest below that, at least in the case of Nicotiana spp., East (1927) has demonstrated that unless the plants under study are "selected advantageously", (our paraphrase) it is the classical model which is incapable of such predictions. East clearly states that the incidence of pseudo self-compatibility is so high as to "make the analysis of results very difficult". Reference is again made to Larsen's (1977) review of others' efforts to force their material into appropriate patterns. Unlike the classical model, the heterosis model predicts and explains the variation in the strength of self-incompatibility reported by many investigators.

Consider now the third point raised by LMC\&F, namely our statement that self-incompatibility results, not from the positive inhibition of pollen tubes but rather because the slow growth of incompatible tubes prevents them from reaching ovules before floral abscission. LMC\&F reject this as a possibility because, in both the species with which they are the most familiar, gynoecia are retained until the end of the flowering season. Floral abscission cannot therefore be implicated in failure of pollen tube growth. We believe that most readers will not be concerned with whether failure of slow growing pollen tubes is due to the loss of stigma, style, pistil, or gynoecium.

LMC\&F also state in their discussion that, if the heterosis, "...model were true, it would, of course be impossible to discover any species with a one-locus system of self-incompatibility". An examination of our fig. 4 will show that this statement is incorrect.

Questioning the classical oppositional model seems all the more reasonable in view of an early paper of self-incompatibility by East (1927). Referring to self-incompatibility in Nicotiana, East stated:

"The reason why these studies ought to prove helpful to others is because the results are comparatively uncomplicated by extraneous variables, due to the fact that in strains of Nicotiana used, the differences between compatible matings is complete, i.e., under ordinary circumstances incompatible matings produce no seeds. Such material is unusual. Ordinarily the block to fertilisation which produces the difference between an incompatible and a compatible union is incomplete.... [With compatible matings] ... fertilisation occurs in about 3 days. Clearly any conditions which extend the "life" of the flower or accelerate the rate of pollentube growth in an incompatible mating, tend to promote fruitfulness in such unions; and I have called such fruitfulness "pseudofertility". In practice, "pseudo-fertile" unions can be obtained with most of the self-sterile strains of Nicotiana used by pollinating the young bud. But unfortunately, material which 
is serviceable for self-sterility studies is often so "pseudo-fertile" under ordinary conditions that an analysis of the results is very difficult without a good working hypothesis as a guide. This is because the average difference in time required for incompatible pollen-tubes and for compatible pollen-tubes to reach the micropyle is so small that the frequency distributions of the two types overlap each other.

$B y$ selecting the material advantageously and by growing it under such favorable conditions that pseudo-fertility is eliminated, it has been possible to isolate seven multiple allelomorphs which control the behavior of self-sterile plants in crosses with each other. These allelomorphs have been termed S1, S2 ... etc. No second locus for self-sterility has been found. One cannot say, of course, that no other loci exist; but if such is the case, the plants with which we have dealt are homozygous for these loci." [According to the heterosis model, individuals heterozygous at other loci would probably exhibit "pseudo-fertility" and thus be excluded from the "advantageously selected material."]

Italics and material within square brackets are ours.

East would not have been surprised that Williams et al., (1983) found that in Lycopersicon peruvianum, another classical example of gametophytic self-incompatibility, 1 out of the 5 plants studied appeared to be pseudo self-compatible, at least to the extent that pollen tubes reach the base of the style. In other cases, Lolium spp., Papaver spp. and Trifolium spp. (Professor J. Brewbaker, pers. comm.) the classical expectations are apparently obtained from the outset. However, ample evidence suggests that, in still others, this is certainly not the case.

LMC\&F state that some of the most convincing evidence on the question of complementary models of self-incompatibility, comes from a recent investigation of autotetraploid ryegrass whose pollen, being diploid etc. We will happily respond when the paper becomes available. In the meantime, we close by emphasising that our primary purpose in presenting the heterosis model was to indicate our opinion that the classical interpretation of gametophytic self-incompatibility needs a careful re-examination. If this were not the case, plant breeders would long ago have transferred self-incompatibility into crop species for the purposes of producing $F_{1}$ hybrids. That this has not been possible to do (Martin, 1961; Rick, 1982), suggests to us that the classical model is not as satisfying as many seem to believe.
Acknowledgements We wish to express sincere thanks to Professors E. Ottaviano and A. Camussi, Institute of Genetics University of Milan, an anonymous reviewer, and also to Nathaniel Mulcahy, for help with different aspects of this paper. Supported, in part, by NSF Grant BSR-8407472.

\section{REFERENCES}

ATWOOD, S. S. 1944. Oppositional alleles in natural populations of Trifolium repens. Genetics, 29, 428-435.

BREWBAKER, J. L. 1954. Incompatibility in autotetraploid Trifolium repens. I. Competition and self-compatibility. Genetics, 39, 307-316.

CAMPBELL, J. M. AND LAWRENCE, M. J. 1981. The population genetics of self-incompatibility polymorphism in Papaver rhoeas. I. The number and distribution of $S$-alleles in families from three localities. Heredity, 45, 69-79.

EAST, E. M. 1927. Peculiar genetic results due to active gametophytic factors. Hereditas, 9, 49-58.

EMERSON, S. 1939. A preliminary survey of the Oenothera organensis population. Genetics, 24, 524-537.

EMERSON, S. 1940. Growth of incompatible pollen tubes in Oenothera organensis. Bot. Gaz., 101, 890-911.

FEARON, C. H., HAYWARD, M. D. AND LAWRENCE, M. J. 1983. Self-incompatibility in ryegrass. V. Genetic control, linkage, and seed-set in diploid Lolium multiflorum Lam. Heredity, 50, 35-45.

GASTEL, A. J. G. VAN AND NETTANCOURT, D. DE. 1975. The generation of new incompatibility alleles. Incompatibility Newsletter, 6, 66-69.

HOFFMAN, F. AND WENZEL, G. 1981. Self-compatibility in microspore-derived doubled-haploid rye lines and single grain selection for alkylresorcinol content. Theor. Appl. Genet., 60, 129-133.

LARSEN, K. 1977. Self-incompatibility in Beta vulgaris L. I. four gametophytic, complementary $S$-loci in sugar beet Hereditas, $85,227-248$.

LAWRENCE, M. J., MARSHALL. D. F., CURTIS, V. E. ANI) FEARON, C. H. 1985. Gametophytic self-incompatibility re-examined: a reply. Heredity, 54, 000-000.

LEWIS, D. 1954. Comparative incompatibility in angiosperms and fungi. Adv, in Genetics, 6, 235-285.

LEWIS, D. AND CROWE, L. K. 1958. Unilateral incompatibility in flowering plants. Heredity, 12, 233-256.

LUNDQVIST, A. 1958. Self-incompatibility in rye. IV. Factors related to self-seeding. Hereditas, 44, 193-256.

MARTIN, F. W. 1961. The inheritance of self-incompatibility in hybrids of Lycopersicon esculentum Mill. $\times$ L. chilense DUN. Genetics, 46, 1443-1454.

MULCAHY, D. L. AND MULCAHY, G. B. 1983. Gametophytid self-incompatibility reexamined. Science, 220, 1247-1251.

NETTANCOURT, D. DE. 1977. Incompatibility in Angiosperms. Springer Verlag, Berlin.

RiCK, C. M. 1982. The potential of exotic germplasm for tomato improvement. In Vasil, I. K., Scowcroft, W. R. and Frey, K. J. (eds.) Plant Improvement and Somatic Cell Genetics, Academic Press, New York, pp. 1-28.

SREE RAMULU, K. 1982. Failure of obligate inbreeding to produce new $S$-alleles in Lycopersicon peruvianum Mill Incompatibility Newsletter, 14, 103-110.

WHITEHOUSE, H. L. K. 1950. Multiple-allelomorph incompatibility of pollen and style in the origin of the angiosperms. Ann. Botan. N. S. 14, 198-216. 
WILliams, E. G., O'NEILL, P. AND HOUGH, T. 1982. The pollen tube as an experimental system. In Williams, E. G., Knox, R. B., Gilbert, J. H., and Bernhardt. P. (eds.) Pollination '82, University of Melbourne, pp. 15-26.

WILLIAMS, w. 1947. Genetics of red clover (Trifolium pratense L.) compatibility. III J. Genet., 48, 69-79.
WRIGHT, s. 1939. The distribution of self-sterility alleles in populations. Genetics, 24, 538-552.

WRIGHT, S. 1964. The distribution of self-sterility alleles in populations. Evolution, 18, 609-619. 